180 Elbs: Beiträge z. Kenntniss aromatischer Ketone.

3) Die Melanurensäure reagirt auf Fünfach-Chlorphosphor unter Bildung von Phosphoroxychlorid und Cyanurchlorid.

4) Gegen sonstige Agentien verhält sich die Säure sehr indifferent.

Leipzig, im August 1885.

\title{
Beiträge zur Kenntniss aromatischer Ketone.
}

Erste Mittheilung;

von

\section{Karl Eibs.}

Bei Gelegenheit der Untersuchungen über Triphenylmethan und einige seiner Homologen bedurfte ich ausser der gewöhnlichen Methode zur Gewinnung von Kohlenwasserstoffen der Triphenylmethangruppe - Einwirkung von Chloroform oder Chlorpikrin auf aromatische Kohlenwasserstoffe in Gegenwart von Aluminiumchlorid - noch andere Verfahren, weil das ersterwähnte nur solche homologe Triphenylmethane liefert, welche am Methankohlenstoffatom 3 gleiche aromatische Reste gebunden enthalten. Fon den vielen bekannten Darstellungsweisen erschienen folgende praktisch brauchbar:

1. Die Umsetzung von Benzalchlorid mit aromatischen Kohlenwasserstoffen bei Gegenwart von Zinkstaub oder Aluminiumchlorid. ${ }^{1}$ )

$$
\mathrm{C}_{6} \mathrm{H}_{5} . \mathrm{CHCl}_{2}+2 \mathrm{O}_{6} \mathrm{H}_{6}=\mathrm{C}_{6} \mathrm{H}_{5} \cdot \mathrm{CH}\left(\mathrm{C}_{6} \mathrm{H}_{5}\right)_{2}+2 \mathrm{HCl} \text {. }
$$

2. Die Condensation secundärer aromatischer Alkohole mit aromatischen Kohlenwasserstoffen mittelst Phosphorpentoxyd.")

$$
\begin{aligned}
& \mathrm{C}_{6} \mathrm{H}_{5} \cdot \mathrm{CHOH} \cdot \mathrm{C}_{6} \mathrm{H}_{5}+\mathrm{C}_{6} \mathrm{H}_{6}=\mathrm{C}_{6} \mathrm{H}_{5} \cdot \dot{\mathrm{C}} \mathrm{H} \cdot \mathrm{C}_{6} \mathrm{H}_{5}+\mathrm{H}_{2} \mathrm{O} . \\
& \text { 1) Böttinger, Ber. Berl. chem. Ges. 12, } 976 . \\
& \text { 2) Baeyer u. Hemilian, das. 7, 1204. }
\end{aligned}
$$


Elbs: Beiträge z. Kenntniss aromatischer Ketone. 181

3. Die Zersetzung der $\beta$-Pinakoline durch Alkalien. ${ }^{1}$ ) $\left(\mathrm{C}_{6} \mathrm{H}_{5}\right)_{3} \cdot \mathrm{C} . \mathrm{CO} . \mathrm{C}_{6} \mathrm{H}_{5}+\mathrm{H}_{2} \mathrm{O}=\left(\mathrm{C}_{6} \mathrm{H}_{5}\right)_{3} \cdot \mathrm{CH}+\mathrm{C}_{6} \mathrm{H}_{5} . \mathrm{COOH}$.

Die erste Synthese erwies sich der schlechten Ausbeute wegen als ungeeignet. Günstigere Resultate lieferten die beiden andern. Das ursprüngliche Ausgangsmaterial für dieselben bildet das Benzophenon und seine Homologen. Zur Gewinnung dieser Ketone konnte nur die FriedelCrafts'sche Synthese in Betracht kommen, welche schon vielfach dazu gedient hatte, jedoch ohne dass bisher in allgemeinerer Weise die günstigsten Versuchsbedingungen festgestellt und genaue, ins Einzelne gehende Angaben daruiber bekannt gemacht wurden. Das nächste Ziel der Untersuchung war also, für die Darstellung der aromatischen Ketone mittelst der Reaction von Friedel und Crafts ein Verfahren auszuarbeiten, welches in weiterem Umfange zuverlässig brauchbar war. Dieses Ziel liess sich in befriedigender Weise erreichen, und da in Folge dessen eine Reihe von Ketonen in grösserer Menge zur Verfügung standen, so wurden dieselben nicht lediglich in oben erwähntem Sinne zur Synthese von Homologen des Triphenylmethans verwendet, sondern auch, sofern sie wenig oder gar nicht bekannt waren, anderweitig untersucht, so betreffs ihrer Fähigkeit, durch Condensation unter Wasseraustritt homologe Anthracene zu bilden.

\section{Verfahren zur Darstellung der Homologen des Benzophenons.}

Kohlenwasserstoff und Säurechlorid werden in äquivalenten Mengen gemischt und nöthigenfalls so viel Schwefelkohlenstoff zugesetzt, dass eine klare Lösung entsteht. In einen geräumigen, mit Rückflusskühler versehenen Kolben giesst man eine dem Volumen obiger Mischung entsprechende Menge Schwefelkohlenstoff, trägt ungefähr so viel Chloraluminium ein, als das Gewicht des angewandten Säurechlorids ausmacht und giebt durch den Kühler in kleinen Mengen das Gemisch zu, indem man jeweils abwartet, bis

1) Thörner u. Zinke, Ber. Berl. chem. Ges. 10, 1475; 11, 65. 
182 Elbs: Beiträge z. Kenntniss aromatischer Ketone.

die unter starker Salzsäureentwicklung und freiwilliger Erwärmung vor sich gehende Reaction nachgelassen bat. Wenn alles eingetragen ist, so erwärmt man auf dem Wasserbade, bis die Salzsäureentwicklung nahezu aufhört. Nach dem Erkalten giesst man durch den Kühler sehr wenig Wasser hinab und schüttelt um; nach Ablauf der stürmischen Einwirkung wiederholt man den Wasserzusatz, bis keine Reaction mehr stattfindet und destillirt hierauf mit Wasserdampf. Dadurch zersetzt sich allenfalls noch vorhandenes Säurechlorid; Schwefelkohlenstoff und noch unangegriffener Kohlenwasserstoff gehen vollständig, die aus dem Säurechlorid entstandene Säure je nach Umständen ganz oder theilweise über, während die Homologen des Benzophenons, welche nur spurenweise mit Wasserdämpfen flüchtig sind, als braungefärbte, schwere Oele oder gelbe, butterartige Massen zurückbleiben. Man giesst die überstehende wässerige Chloraluminiumlösung $a b$, so gut es angeht, setzt sehr verdünnte rohe Salzsäure zu und leitet nochmals $1 / 2$ Stunde lang Dampf ein; dann wird die wässrige Flüssigkeit wieder abgegossen, das Keton mit Wasser gewaschen, sehr verdünnte Natronlauge zugegeben, wiederum $1 / 2$ Stunde lang Dampf eingeleitet und mit Wasser gewaschen. Man trennt nun das Keton im Scheidetrichter vom Wasser, erwärmt es, um alle Feuchtigkeit zu vertreiben, kurze Zeit auf $110^{\circ}$ und unterwirft es der fractionirten Destillation. In Fällen, wo das Vorhandensein von Isomeren ausgeschlossen ist, bleibt schon bei der ersten Destillation der Siedepunkt fast ganz constant. Die Ausbeute an reinen Ketonen beträgt 50\% $-80 \%$ der berechneten.

Alle bei der Ausführung der Synthese in Anwendung kommenden Gefässe und Substanzen müssen natürlich gut getrocknet sein. Um während der Dạrstellung das Eindringen von Feuchtigkeit aus der Atmosphäre zu verhindern, empfiehlt es sich, in das Gasleitungsrohr, welches aus dem obern Ende des Kühlers den Chlorwasserstoff wegführt, ein Chlorcalciumrohr einzuschalten.

Die vortheilhafte Wirkung des Schwefelkohlenstoffs ist eine dreifache; fürs erste dient er als Verdünnungsmittel, 
welches eine langsame, ruhige Umsetzung sichert; dann hält er beim Erwärmen auf dem Wasserbade die Temperatur stets in der für die Reaction günstigsten Höhe von etwa $50^{0}$ und schliesslich verhindert er, dass bei der heftigen Einwirkung des Wassers auf die Reactionsmasse ein Theil der letzteren verharzt; specielle Versuche, wobei vor dem Wasserzusatz der Schwefelkohlenstoff abdestillirt wurde, zeigten, dass gerade der letzterwähnte Umstand recht wesentlich ist, zumal neben der Verharzung der Ketone noch eine theilweise Zersetzung eintritt. Reine Ketone, mit Chloraluminium gemischt und mit Wasser versetzt, liefern neben Harzen mehr oder weniger Kohlenwasserstoff und Säure, indem beispielsweise nach folgender Gleichung Umsetzung stattfindet:

$\mathrm{C}_{6} \mathrm{H}_{3}\left(\mathrm{CH}_{3}\right)_{2}$. OO. $\mathrm{C}_{6} \mathrm{H}_{3}+\mathrm{H}_{2} \mathrm{O}=\mathrm{C}_{6} \mathrm{H}_{4}\left(\mathrm{CH}_{3}\right)_{2}+\mathrm{C}_{6} \mathrm{H}_{5} \cdot \mathrm{COOH}$.

Die Menge des Aluminiumchlorids lässt sich in manchen Fällen bis auf die Hälfte vom Gewichte des angewandten Säurechlorids ermässigen ohne Beeinträchtigung der Ausbente. Die zur Umsetzung nöthige Zeit wechselt von $1 / 2$ bis zu 2 Tagen. Wenn die Reaction, wie es mitunter vorkommt, in der Kälte nicht sofort von selbst eintritt, so genügt eine geringe Erwärmung, um sie einzuleiten.

Ein Auskochen des Rohproduktes mit verdünnter Salzsäure ist nothwendig, um die Ketone frei von Thonerde zu erhalten; die ursprüngliche Reactionsmasse scheint nämlich die Ketone grösstentheils nicht im freien Zustande, sondern als Chloraluminiumverbindungen $\mathrm{zu}$ enthalten, welche allerdings sehr unbeständig sind, aber nur schwer sich vollkommen zersetzen; dazu kommt noch, dass ausgeschiedenes Thonerdehydrat mit der organischen Substanz sich zu einer emulsionsartigen Masse innig mischt, durch die Salzsäure jedoch leicht in Lösung gebracht wird. Unterwirft man ein Keton, ohne es vorher mit Salzsäure ausgekocht zu haben, der Destillation, so tritt unter Thonerdeabscheidung eine mehr oder minder beträchtliche Verkohlung ein, wodurch die Ausbente verringert und die Reindarstellung erschwert wird. Manche Homologe des Benzophenons werden durch 
184 Elbs: Beiträge z. Kenntniss aromatischer Ketone.

heisse Salzsäure etwas angegriffen, weshalb man diese sehr verdünnt nehmen muss und das Kochen damit nicht allzu lange fortsetzen darf. Das nämliche gilt für die Natronlauge, welche man in vielen Fällen zweckmässig durch Sodalösung ersetzt. Der Zusatz von Alkali dient zur vollständigen Entfernung der aus den Säurechloriden herrührenden organischen Säuren, was sich durch blosse Destillation mit Dampf nur sehr schwer erreichen lässt; bei der Darstellung symmetrischer Ketone mittelst Phosgen unterbleibt er selbstverständlich.

Was den Umfang der Brauchbarkeit dieser eben beschriebenen Darstellungsmethode anbelangt, so gibt sie sehr gute Resultate bei Benzol und seinen Homologen, weniger gute bei den complicirteren aromatischen Kohlenwasserstoffen, wo sie mitunter ganz im Stiche lässt. Im Kern halogenisirte Kohlenwasserstoffe reagiren schlecht, Nitrokörper gar nicht; im Molekül des Säurechlorids eingelagerte negative Atome oder Gruppen beeinträchtigen dagegen die Umsetzung gewöhnlich nicht.

Reduction der aromatischen Ketone zu secundären Alkoholen.

Die Verwendung von Natriumamalgam ist zur Ueberführung grösserer Mengen von Ketonen in die entsprechenden Alkohole unbequem und kostspielig; es wurde desshalb als Reductionsmittel nach dem Vorgange von $Z_{\text {agumenny }}{ }^{1}$ ) Zinkstaub und Kali angewendet. Folgendes Verfahren hat sich bewährt: 1 Theil Keton wird in 10-20 Theilen Weingeist gelöst und 5-10 Theile Zinkstaub nebst einigen Cubikcentimetern sehr concentrirter wässriger Kalilauge zugegeben. Man lässt es einige Tage an einem warmen Orte stehen unter zeitweiligem Umschütteln und Zusatz von ein paar Tropfen Kalilauge. Erwärmung auf dem Wasserbade am Rückflusskübler beschleunigt zwar die Reduktion, ist aber unnöthig und insoforn nachtheilig, als leicht Verunreinigung des Produktes durch braunes Harz eintritt. Um den Alko-

1) Zagumenny, Ann. Chem. Pharm. 184, 174. 
hol rein zu gewinnen, giesst man die klare Lösung rom Zinkstaub $a b$, wäscht denselben noch mit etwas Weingeist aus und versetzt mit viel Wasser; das ausgefällte Carbinol wird destillirt oder umkrystallisirt. Da manche dieser Alkohole in Wasser merklich löslich sind, so erleidet man bei dem eben besprochenen Verfabren einen Verlust. Es lässt sich dies vermeiden, wenn man in die ursprüngliche Lösung Kohlensäure einleitet, wodurch der grösste Theil des Kalis und alles Zink als Carbonate ausgefällt werden, dann filtrirt und krystallisiren lässt oder destillirt. Man gewinnt $60^{\%} / 0$ bis $80 \%$ der berechneten Menge an reinem Alkohol.

Die Condensation der secundären Alkohole mit aromatischen Kohlenwasserstoffen zu homologen Triphenylmethanen

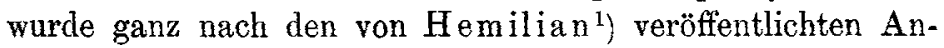
gaben ausgeführt.

\section{Darstellung der $\beta$-Pinakoline.}

Die Darstellung der $\beta$-Pinakoline geschah nach der von Thörner ${ }^{2}$ ) zur Gewinnung des $\beta$-Phenyltolylpinakolins gegebenen Vorschrift durch Kochen der Ketone mit Zink und alkoholischer Salzsäure während mehrerer Tage. Es bilden sich bei dieser Reduction ziemlich viele Nebenprodukte und da die $\beta$-Pinakoline nicht unzersetzt destillirbar sind, auch meistens nur geringe Krystallisationsfähigkeit besitzen, so ist es manchmal schwer, sie rein zu erhalten. Aus diesem Grunde stellt man Kohlenwasserstoffe der Triphenylmethangruppe fast ausnahmslos bequemer dar durch Condensation secundärer aromatischer Alkohole als durch Spaltung von $\beta$-Pinakolinen.

Synthesevon Anthracenen durch Wasserabspaltung aus aromatischen Ketonen.

Unsre Kenntniss der Homologen des Anthracens ist bis jetzt noch eine ziemlich lüokenhafte; es rührt dies daher, dass dieselben im Steinkohlentheer anscheinend nur in

1) Hemilian, Ber. Berl. chem. Ges. 7, 1204.

2) Thörner, Ann. Chem. Pharm. 189, 110. 
186 Elbs: Beiträge z. Kenntniss aromatischer Ketone.

geringen Mengen auftreten, zu ihrer Grewinnung auf synthetischem Wege aber in weiterem Umfange praktisch brauchbare Methoden fehlen. Das Verfahren von Liebermann und Tobias ${ }^{2}$ ) - Wasserentziehung aus homologen Hydroanthranolen - liefert ausschliesslich Anthracene mit einer Seitenkette in $\gamma$-Stellung. Eine Reihe anderer Bildungsweisen - durch Erhitzen in der Seitenkette halogenisirter Kohlenwasserstoffe unter Druck $^{2}$ ), durch Einwirkung von Halogenmethanen auf aromatische Kohlenwasserstoffe bei Gegenwart von Chloraluminium ${ }^{3}$, durch $W$ asserstoffabspaltung aus homologen Diphenylmethanen ${ }^{4}$ ) - eignen sich wenig als Darstellungsmethoden, weil sie nur in einzelnen Fällen ausführbar sind und gewöhnlich nur geringe Ausbeute liefern.

Aus diesem Grunde war es von Interesse, Versuche anzustellen, ob nicht eine von Behr und Dorp ${ }^{5}$ ) aufgefundene Reaction sich verallgemeinern lasse. Diese Forscher machten die für Aufklärung der Struktur des Anthracens wichtige Beobachtung, dass das o-Tolylphenylketon beim Erhitzen unter Wasserbildung in Anthracen übergehe nach folgender Gleichung:

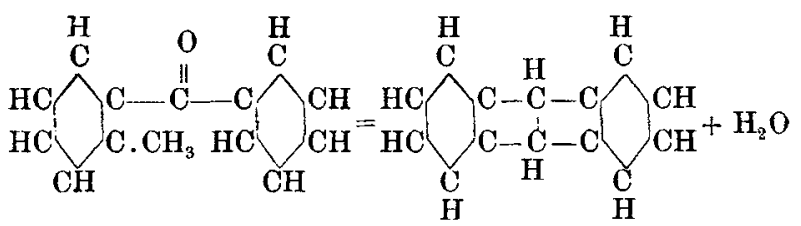

Man konnte erwarten, dass eine derartige Condensation bei allen solchen Homologen des Benzophenons möglich sei, welche eine Methylgruppe in Orthostellung zur Carbonylgruppe enthalten. Traf diese Voraussetzung ein, so war

1) Liebermann u. Tobias, Ber. Berl. chem. Ges. 14, 795.

2) Dorp, Ann. Chem. Pharm. 169, 210.

${ }^{3}$ ) Anschütz u. Romig, Ber. Berl. chem. Ges. 18, 664. Elbs u. Wittich, das. 18,348 .

4) Weilex, das. 7, 1185. Fischer, das. 7, 1195.

5) Behr u. Dorp, das. 7, 17. 
Elbs: Beiträge z. Kenntniss aromatischer Ketone. 187 damit ein Weg gegeben, um von leicht zu beschaffenden aromatischen Ketonen zu bomologen Anthracenen zu gelangen, deren Struktur überdies kanm zweifelhaft war, da man von dem als Ausgangsmaterial dienenden Keton her die Stellung der Seitenketten kannte, und eine Umlagerung derselben bei der Wasserabspaltung durch einfache Erhitzung unwahrscheinlich schien.

Die bis jetzt angestellten, zum Theil schon kurz veröffentlichten Untersuchungen ${ }^{1}$ ) lassen noch keinen sichern Schluss darüber $z u$, innerhalb welcher Grenzen und unter welchen Umständen die besprochene Synthese homologer Anthracene allgemein ausführbar ist. Denn während die Condensation in einzelnen Fällen leicht vor sich geht, gelingt sie in andern nicht, ohne dass sich ein Grund dafür erkennen lässt. Bei solchen Ketonen, welche durch blosses Erhitzen keine Anthracenbildung zeigen, lässt sich dieselbe auch nicht herbeiführen durch Condensationsmittel. Ohne Erfolg wurden als solche versucht: concentrirte Schwefelsäure, saures schwefelsaures Kalium, Phosphorpentoxyd, Chlorzink.

Zur Ausführung der Condensation erhitzt man die Ketone in kleinen, langhalsigen Kolben $3-6$ Tage lang bis eben zum Sieden. Dann wird die feste, schwarzbraune Masse mit siedendem Alkohol ausgezogen, wobei sich allmählich alles löst bis auf einen geringen verkohlten Risckstand; die Anthracene erhält man aus der alkoholischen Lösung gewöhnlich als hellbraune krystallinische Pulver oder Krusten, welche zur Reinigung aus Schwefelkohlenstoff oder Benzol umkrystallisirt werden; am schnellsten gewinnt man kleine Mengen reiner Substanz durch vorsichtige Sublimation der unreinen, aus Alkohol abgeschiedenen Krystalle. Das Rohprodukt zu sublimiren ist unvortheilhaft, weil der grösste Theil dabei verkohlt. Die ursprünglichen alkoholischen Mutterlaugen hinterlassen beim Eindunsten beträchtliche Mengen dicker brauner Oele, welche der Hauptsache nach

1) Elbs u. Larsen, Ber. Berl. chem. Ges. 17, 2848. Claus u. Elbs, das. 18, 1797. 
188 M@ Gowan: Ueber einige Abkömmlinge des

aus unveränderten Ketonen bestehen; man verarbeitet sie am besten ohne weitere Reinigung durch nochmaliges Erhitzen auf Anthracene.

Weitere Mittheilungen werden die speciellen Resultate der hauptsächlich in den angedeuteten Richtungen an einer Reihe von Homologen des Benzophenons ausgeführten Untersuchungen enthalten.

Freiburg, im Januar 1886.

\section{Ueber einige Abkömmlinge des Schwefelharnstoffes;}

von

\section{G. MI Gowan.}

Im Anschluss an meine Arbeit ,über Abkömmlinge der Methylsulfonsäure etc." $\left.{ }^{1}\right)$ habe ich, angeregt durch eine Mittheilung des Hrn. O. Loew, Schwefelharnstoff auf Trichiormethylsulfonchlorid einwirken lassen. Dadurch wurde ich zum Studium einiger Schwefelharnstoff-Verbindungen geführt.

Wird zu einer wässrigen Lösung von Schwefelharnstoff obiges Chlorid gefügt, so findet in der Kälte keine Reaction statt; in der Wärme aber löst sich dasselbe allmählich unter Abscheidung von Schwefel auf. Wenn dagegen eine kühle Lösung des Schwefelharnstoffs in 80-90 procent. Alkohol mit jenem Chlorid versetzt wird, so scheiden sich unter Wärmeentwickelung reichlich Nadeln aus, deren Menge durch Zusatz von Aether vermehrt wird.

Diese Verbindung ist identisch mit dem von $\mathrm{Claus}^{2}$ ), durch Einwirkung von Chlor auf Schwefelharnstoff erhaltenen Dischwefelharnstoffdichlorid: $\left(\mathrm{CSN}_{2} \mathrm{H}_{4}\right)_{2} \mathrm{Cl}_{2}$. Aus Trichlormethylsulfonchlorid stellt man dasselbe am besten durch allmähliches Zufügen ron reinem Chlorid (1 Mol.) zu der

1) Dies Journ. [2] 30, $280 \mathrm{ff}$.

2) Ann. Chem. Pharm. 179, 139. 